\title{
Analytical Study of Radiative Casson Nanoliquid Flow with Heat Absorption
}

\author{
K. Loganathan ${ }^{1(\bowtie)}$, K. Tamilvanan ${ }^{2}$, Amelec Viloria ${ }^{3,4}$, Noel Varela ${ }^{4}$, \\ and Omar Bonerge Pineda Lezama ${ }^{5}$ \\ 1 Department Mathematics, Faculty of Engineering, \\ Karpagam Academy of Higher Education, Coimbatore 641021, Tamilnadu, India \\ loganathankaruppusamy304@gmail .com \\ 2 Department of Mathematics, Government Arts College for Men, \\ Krishnagiri 635001, Tamilnadu, India \\ 3 Universidad de la Costa, Barranquilla, Colombia \\ 4 Universidad Peruana de Ciencias Aplicadas, Lima, Peru \\ ${ }^{5}$ Universidad Tecnolgica Centroamericana (UNITEC), San Pedro Sula, Honduras
}

\begin{abstract}
The divergence of thermally radiative MHD flow of a Casson nanofluid over a stretching paper alongside heat absorption. The governing non linear equations are remodeled into a nonlinear ODE's. The HAM is adopted to find the series solution. The changes of pertinent parameters are analyzed with diagrams and tables. The fluid velocity is controlled by suction and it develops with injection. The local Nusselt number rapidly suppresses with increasing the magnetic field parameter in heat generation case.
\end{abstract}

Keywords: Casson nanoliquid · Heat absorption · Magnetic field · Thermal radiation

\section{Introduction}

Most of the engineering and industrial processes depend on heat transfer mechanism, because they have cooling and heating processes. In general, the ordinary fluids are transfer less amount heat because they owing poor thermal conductivity. Various researchers are tried to increase the fluid thermal conductivity in different ways. One of the simplest method is nanosized particles are suspended into an ordinary fluids to raise the fluid thermal conductivity. Applications of nanofluids are investigated by many authors for both the nanofluids with Newtonian or non-Newtonian base with different geometrical shapes. One of the base fluid is Casson fluid and which posses yield stress. After applying the shear stress, Casson fluid performs as a solid when low shear stress and it moves when higher shear stress compared to the yield stress. Example of these fluids are soup, blood, jelly, tomato sauce, etc. Some important studies in this directions are $[1-10]$. 


\section{Governing Equations}

The flow system is modeled with

1. Incompressible flow

2. Casson nanoliquid

3. thermal radiation

4. Magnetic field

5. Buongiorno nanofluid model

6. Stretching sheet with linear velocity.

7. heat absorption

$$
\begin{aligned}
& \frac{\partial u}{\partial x}+\frac{\partial v}{\partial y}=0 \\
& u \frac{\partial u}{\partial x}+v \frac{\partial u}{\partial y}=v\left(1+\frac{1}{\beta}\right) \frac{\partial^{2} u}{\partial y^{2}}-\left(\frac{\sigma B_{n}^{2}}{\rho}\right) \\
& u \frac{\partial T}{\partial x}+v \frac{\partial T}{\partial y}=\alpha_{T} \frac{\partial^{2} T}{\partial y^{2}}-\frac{16 \sigma_{s} T_{\infty}^{2}}{3 k_{e} \rho C_{p}}+\frac{\rho^{*} C_{p}^{*}}{\rho C_{p}}\left[D_{B} \frac{\partial C}{\partial y} \frac{\partial T}{\partial y}+\frac{D_{T}}{T_{\infty}} \frac{\partial T^{2}}{\partial y}\right] \\
&+\frac{Q}{\rho C_{p}}\left(T-T_{\infty}\right) \\
& u \frac{\partial C}{\partial x}+v \frac{\partial C}{\partial y}=D_{B} \frac{\partial^{2} C}{\partial y^{2}}+\frac{D_{T}}{T_{\infty}} \frac{\partial^{2} T}{\partial y^{2}}
\end{aligned}
$$

The boundary points of the above system are:

$$
\begin{array}{r}
u=u_{w}(x)=a x, v=v_{w}, \text { at } y=0 \\
u \rightarrow 0, v \rightarrow 0, T \rightarrow T_{\infty}, C \rightarrow C_{\infty}, \text { at } y \rightarrow \infty
\end{array}
$$

where $\beta$ (=Casson fluid parameter), $\sigma$ (=electrical conductivity), $\rho$ (=density of fluid), $\alpha_{T}$ (=thermal diffusivity), $C_{p}$ (=specific heat), $D_{B}$ (= Brownian diffusion), $D_{T}$ (+ thermophoretic diffusion coefficient), $Q$ (=heat absorption/generation coefficient).

Consider the transformations:

$$
\eta=\sqrt{\frac{a}{\nu}} y, v=-\sqrt{a \nu} f(\eta), u=a x f^{\prime}(\eta), \theta=\frac{T-T_{\infty}}{T_{w}-T_{\infty}} ; \theta=\frac{C-C_{\infty}}{C_{w}-C_{\infty}}
$$

The following ODEs are retreived from the governing system using above transformations,

$$
\begin{aligned}
& \left(1+\frac{1}{\beta}\right) f^{\prime \prime \prime}(\eta)+f^{\prime \prime}(\eta) f(\eta)-f^{\prime 2}(\eta)-M f^{\prime}(\eta)=0 \\
& \left(1+\frac{4}{3} R d\right) \theta^{\prime \prime}(\eta)+\operatorname{Pr} \theta^{\prime}(\eta) f(\eta)+\operatorname{Pr} N b \theta^{\prime}(\eta) \phi^{\prime}(\eta)+\operatorname{Pr} N t \theta^{\prime 2}(\eta) \\
& +\operatorname{PrHg} \theta=0 \\
& \phi^{\prime \prime}(\eta)+S c\left(f \phi^{\prime}\right)+\frac{N t}{N b} \theta^{\prime \prime}(\eta)=0
\end{aligned}
$$


The boundary points of $f, \theta, \phi$ becomes

$$
f(0)=f_{w}, f^{\prime}(0)=1, f^{\prime}(\infty)=0, \theta(\infty)=0, \phi(\infty)=0
$$

where $M=\frac{\sigma B_{n}^{2}}{\rho}$ [=magnetic field parameter $] \operatorname{Pr}=\nu / \alpha_{T}$ [=Prandtl number], $N b=\left(\frac{\rho^{*} c_{p}^{*}}{\rho c_{p}} D_{B}\left(C_{W}-C_{\infty}\right)\right) / \nu$ [=Brownian motion] parameter, $N t=$ $\left(\frac{\rho^{*} c_{p}^{*}}{\rho c_{p}} D_{T}\left(T_{W}-T_{\infty}\right)\right) /\left(\nu T_{\infty}\right)$ [=thermophoresis parameter], $H g=Q / \rho c_{p} a$ [=heat generation/absorption parameter $], S c=\frac{\nu}{D_{B}}$ [= Schmidt number $], R d=$ $\frac{4}{3} \frac{\sigma_{s} T_{\infty}^{3}}{k_{e} \rho c_{p} \alpha_{T}}[$ [=radiation parameter $]$.

The surface drag force and heat transfer rate can be defined as:

$$
\frac{1}{2} C_{f} \sqrt{R e}=f^{\prime \prime}(0)\left(1+\frac{1}{\beta}\right) \text { and } \frac{N u}{\sqrt{R e}}=-\theta^{\prime}(0)\left(1+\frac{4}{3} R d\right)
$$

Table 1. Order of approximations

\begin{tabular}{c|l|l|l}
\hline Order & $-F_{\eta \eta}(0)$ & $-\theta_{\eta}(0)$ & $-\phi_{\eta}(0)$ \\
\hline 1 & -1.875 & -0.229472 & -0.825641 \\
\hline 5 & -2.07069 & -0.235657 & -0.631038 \\
\hline 10 & -2.07751 & -0.234869 & -0.61575 \\
\hline 15 & -2.07737 & -0.235076 & -0.621188 \\
\hline 20 & -2.07737 & -0.235057 & -0.620607 \\
\hline 25 & -2.07737 & -0.235014 & -0.620104 \\
\hline 30 & -2.07737 & -0.235064 & -0.620437 \\
\hline 35 & -2.07737 & -0.235032 & -0.620348 \\
\hline 40 & -2.07737 & -0.235041 & -0.620326 \\
\hline
\end{tabular}

\section{Results and Discussion}

The present nonlinear system was solve through HAM technique. The HAM was computed via MATHEMATICA software. Figure 1 sketched for the convergent solutions of the current study. Appoximation orders of HAM is shown in Table 1. The examinations are complete for various range of the relevant parameters intricate in this study. It is clear from Fig. 2 the velocity profile enhances for magnetic parameter $(M)$ whereas it reduces for suction/ injection parameter 
Table 2. The surface drag force and heat transfer ratevalues of $\beta, F_{w}, \mathrm{M}, \mathrm{Nb}, \mathrm{Nt}, \mathrm{Rd}$, $\mathrm{Hg}$

\begin{tabular}{|c|c|c|c|c|c|c|c|c|}
\hline$\beta$ & $F_{w}$ & $\mathrm{M}$ & $\mathrm{Nb}$ & $\mathrm{Nt}$ & $\mathrm{Rd}$ & $\mathrm{Hg}$ & $\frac{1}{2} C_{f} \sqrt{R e}$ & $\frac{N u}{\sqrt{R e}}$ \\
\hline 0.3 & 0.2 & 0.3 & -0.2 & 0.1 & 0.2 & 0.2 & -2.23314 & -0.329693 \\
\hline 0.1 & & & & & & & -1.96458 & -0.329552 \\
\hline 1.5 & & & & & & & -1.81318 & -0.328395 \\
\hline 0.1 & & & & & & & -1.75965 & -0.327874 \\
\hline \multirow[t]{4}{*}{0.5} & 0.2 & 0.3 & 0.1 & 0.1 & 0.3 & -0.2 & -1.74059 & -0.27505 \\
\hline & -0.5 & & & & & & -1.83052 & -0.312842 \\
\hline & 0.5 & & & & & & -1.92547 & -0.354811 \\
\hline & 0.1 & & & & & & -1.97484 & -0.400887 \\
\hline \multirow[t]{7}{*}{0.5} & 0.2 & 0.3 & 0.1 & 0.1 & 0.3 & -0.2 & -1.83493 & -0.640482 \\
\hline & & 0 & & & & & -1.91934 & -0.633721 \\
\hline & & 0.5 & & & & & -2.15183 & -0.633721 \\
\hline & & 0.1 & & & & & -2.22367 & -0.615345 \\
\hline & & & 0 & & & & -1.87965 & -0.621188 \\
\hline & & & 2 & & & & -1.81318 & -0.610898 \\
\hline & & & 0.1 & & & & -1.75965 & -0.602256 \\
\hline \multirow[t]{4}{*}{0.5} & 0.2 & 0.3 & 0.1 & 0.1 & 0.3 & -0.2 & -0.329107 & -0.791791 \\
\hline & & & & 0 & & & -0.328067 & -0.621188 \\
\hline & & & & 2 & & & -0.327535 & -0.451693 \\
\hline & & & & 0.1 & & & -0.326996 & -0.28316 \\
\hline \multirow[t]{4}{*}{0.5} & 0.2 & 0.3 & 0.1 & 0.1 & 0.3 & -0.2 & -0.434391 & -0.607522 \\
\hline & & & & & 0 & & -0.45963 & -0.636994 \\
\hline & & & & & 2 & & -0.64889 & -0.639523 \\
\hline & & & & & 0.1 & & -0.67117 & -0.64451 \\
\hline \multirow[t]{4}{*}{0.5} & 0.2 & 0.3 & 0.1 & 0.1 & 0.3 & -0.2 & -0.340084 & -0.616351 \\
\hline & & & & & & -0.5 & -0.333235 & -0.627037 \\
\hline & & & & & & 0.5 & -0.329107 & -0.634088 \\
\hline & & & & & & 0.1 & -0.271261 & -0.65124 \\
\hline
\end{tabular}

$\left(f_{w}\right)$. In Fig. 3 temperature $(\theta(\eta))$ increases for the radiation $(R d)$, Brownian constant $(\mathrm{Nb})$, heat absorption constant $(\mathrm{Hg})$, thermophoresis constant $(\mathrm{Nt})$ and Casson parameter $(\beta)$ and it decays for suction/injection parameter $\left(f_{w}\right)$ and Prandtl number $(P r)$. The concentration $(\phi(\eta))$ rises with higher $(\beta)$ and $N t$ but it diminishes with upsurge in $\left(f_{w}\right)$ and $S c$ (see Fig. 4) (Table 2). 


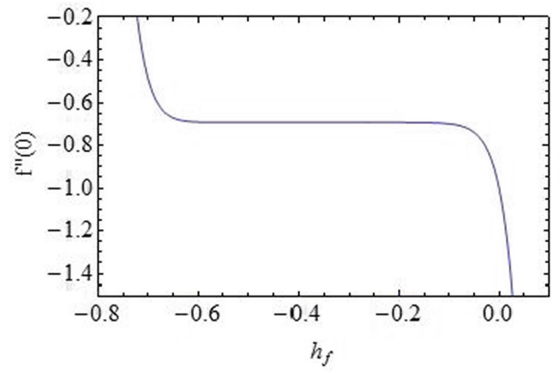

(a)

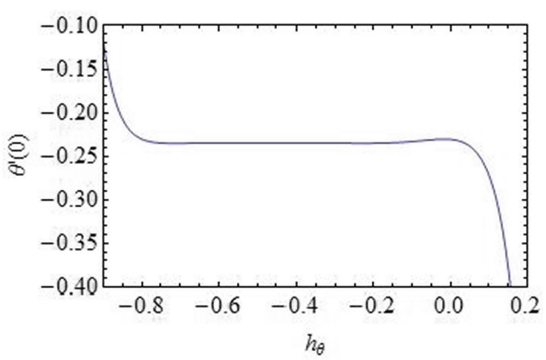

(b)

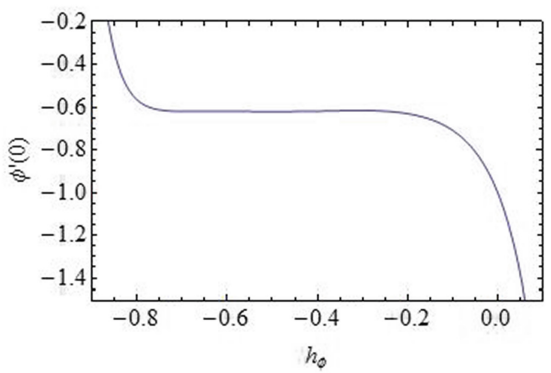

(c)

Fig. 1. h-curves for $h_{f, \theta, \phi}$

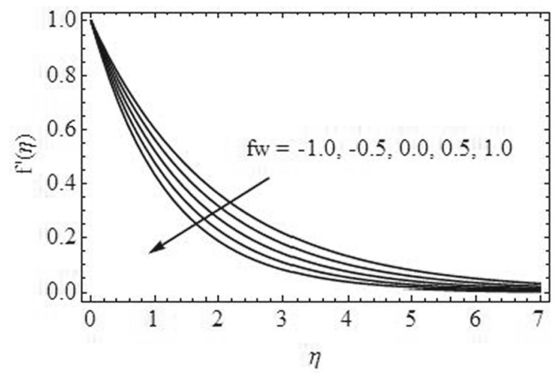

(a)

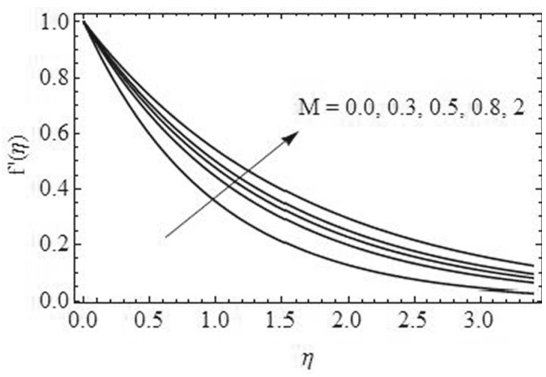

(b)

Fig. 2. Influence of $f_{w}$ and $M$ on velocity 


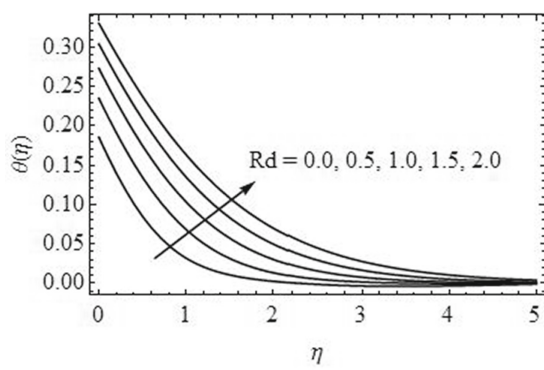

(a)

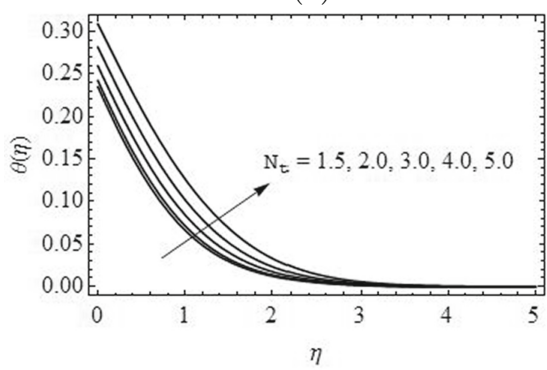

(c)

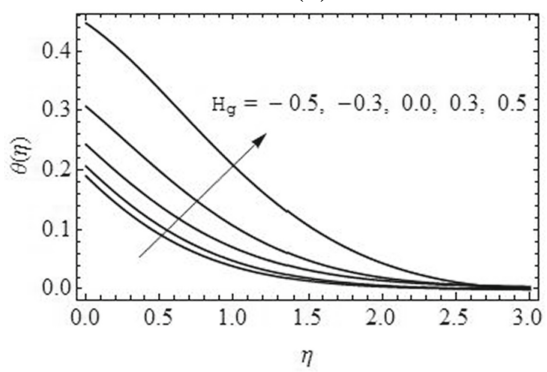

(e)

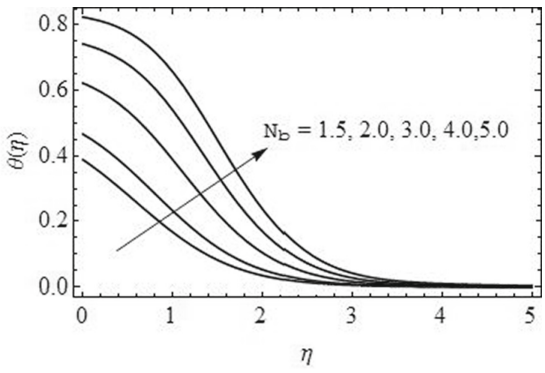

(b)

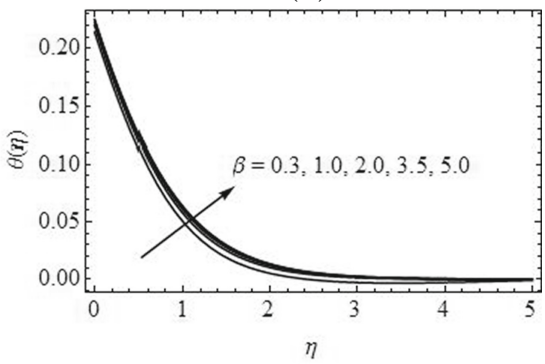

(d)

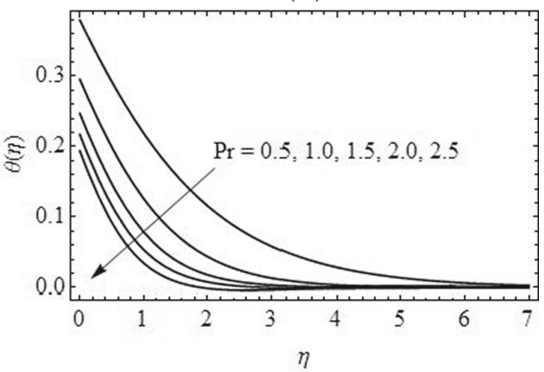

(f)

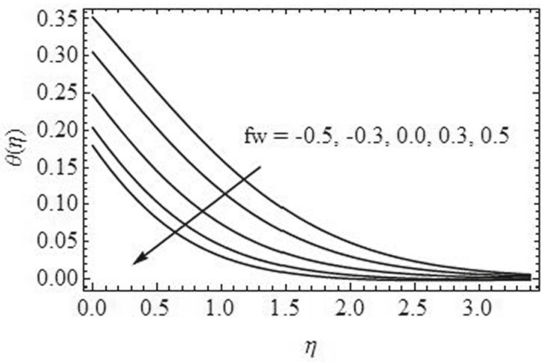

(g)

Fig. 3. Influence of $R d, N b, N t, \beta, H g, P r$ and $f_{w}$ on temperature 


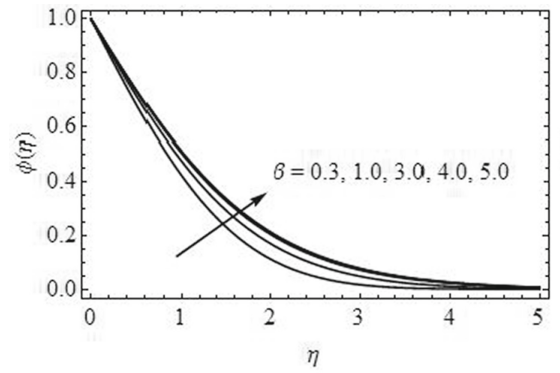

(a)

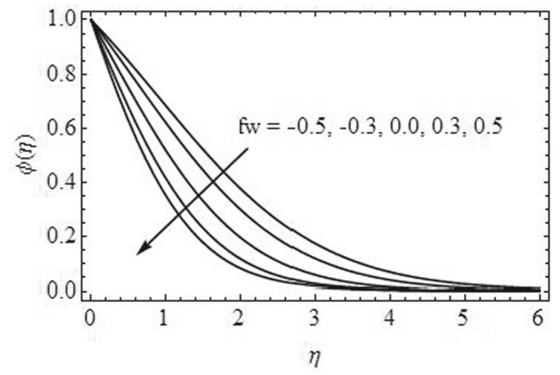

(c)

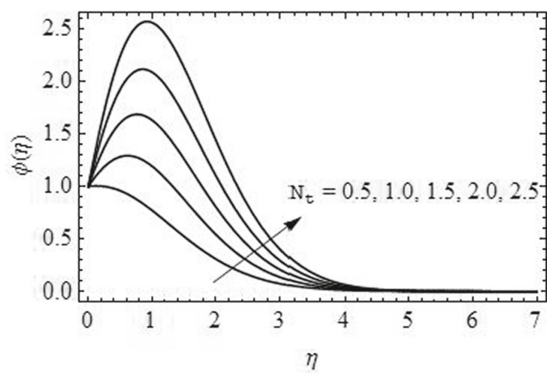

(b)

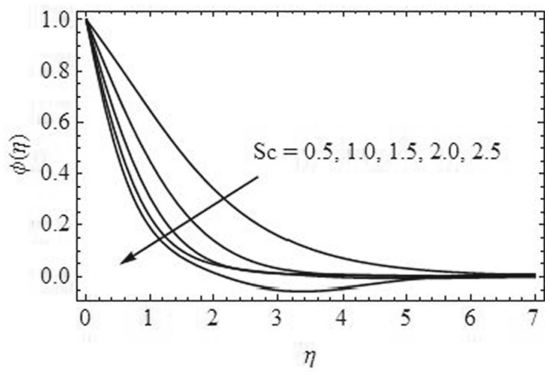

(d)

Fig. 4. Influence of $\beta, N b, f_{w}$ and $S c$ on concentration

\section{Conclusion}

The key features of the present study is given below:

- Temperature profile enhances while increasing $\mathrm{Rd}, \mathrm{Nb}, \mathrm{Nt}$, and $\mathrm{Hg}$.

- Casson parameter $(\beta)$ enhances the concentration and temperature profiles.

- Higher range of Prandtl number (Pr) deduce the thermal boundary.

\section{References}

1. Loganathan, K., Sivasankaran, S., Bhuvaneshwari, M., Rajan, S.: Second-order slip, cross- diffusion and chemical reaction effects on magneto-convection of Oldroyd-B liquid using Cattaneo-Christov heat flux with convective heating. J. Therm. Anal. Calorim. 136, 401-409 (2019)

2. Hayat, T., Imtiaz, M., Alsaedi, A., Almezal, S.: On Cattaneo-Christov heat flux in MHD flow of Oldroyd-B fluid with homogeneous-heterogeneous reactions. J. Magn. Mater. 401(1), 296-303 (2016)

3. Eswaramoorthi, S., Sivasankaran, S., Bhuvaneswari, M., Rajan, S.: Soret and Dufour effects on viscoelastic boundary layer flow over a stretchy surface with convective boundary condition with radiation and chemical reaction. Sci. Iran B. 23(6), 2575-2586 (2016) 
4. Elanchezhian, E., Nirmalkumar, R., Balamurugan, M., Mohana, K., Prabu, K.M.: Amelec Viloria: Heat and mass transmission of an Oldroyd-B nanofluid flow through a stratified medium with swimming of motile gyrotactic microorganisms and nanoparticles. J. Therm. Anal. Calorim. (2020). https://doi.org/10.1007/ s10973-020-09847-w

5. Loganathan, K., Rajan, S.: An entropy approach of Williamson nanofluid flow with Joule heating and zero nanoparticle mass flux. J. Therm. Anal. Calorim. (2020). https://doi.org/10.1007/s10973-020-09414-3

6. Bhuvaneswari, M., Eswaramoorthi, S., Sivasankaran, S., Hussein, A.K.: Crossdiffusion effects on MHD mixed convection over a stretching surface in a porous medium with chemical reaction and convective condition. Eng. Trans. 67(1), 3-19 (2019)

7. Loganathan, K., Sivasankaran, S., Bhuvaneswari, M., Rajan S.: Dufour and Soret effects on MHD convection of Oldroyd-B liquid over stretching surface with chemical reaction and radiation using Cattaneo-Christov heat flux. IOP: Mater. Sci. Eng. 390, 012077 (2018)

8. Bhuvaneswari, M., Eswaramoorthi, S., Sivasankaran, S., Rajan, S., Saleh Alshomrani, A.: Effects of viscous dissipation and convective heating on convection flow of a second-grade liquid over a stretching surface: an analytical and numerical study. Scientia Iranica B 26(3), 1350-1357 (2019)

9. Loganathan, K., Mohana, K., Mohanraj, M., Sakthivel, P., Rajan, S.: Impact of 3rd-grade nanofluid flow across a convective surface in the presence of inclined Lorentz force: an approach to entropy optimization. J. Therm. Anal. Calorim. (2020). https://doi.org/10.1007/s10973-020-09751-3

10. Abbasbandy, S., Hayat, T., Alsaedi, A., Rashidi, M.M.: Numerical and analytical solutions for Falkner-Skan flow of MHD Oldroyd-B fluid. Int. J. Numer. Methods Heat Fluid Flow 24, 390-401 (2014) 